\title{
A case of a young patient diagnosed with the Hippel-Lindau disease c.57I C>G mutation (Croatian form) and multiple paragangliomas after developing ischemic stroke
}

\begin{abstract}
Correlation between erythrocytosis and ischemic stroke is very common and not at least unusual. I present the case of a young patient who was first diagnosed with secondary erythrocytosis and after he developed an ischemic stroke more detailed diagnosis was made. He was then diagnosed with VHL c.571 C $>\mathrm{G}$ mutation (Croatian type). This case shows us the importance of scrutinizing cases, especially in very young patients.
\end{abstract}

Keywords: secondary erythrocytosis, hippel-lindau disease, c.571 C>G mutation, multiple paraganglioma, stroke
Volume I I Issue I - 202 I

\author{
Katarina Blazina \\ Department of Neurology, Clinical Hospital Centre Zagreb, \\ Croatia \\ Correspondence: Katarina Blazina, Department of Neurology, \\ Clinical Hospital Centre Zagreb, \\ Croatia,Tel 0038599 249| 104,Email katarinabl@gmail.com
}

\section{Introduction}

Erythrocytosis is one of the major risk factors for the ischemic cerebrovascular disease. ${ }^{1}$ In cases of the young-onset disease, we always have to keep in mind possible genetic mutations ( and it's subtypes) that have been identified over the last several years: VHL, RET, SDHB, SDHD. ${ }^{2}$ The Hippel-Lindau disease ( VHL) is usually associated with multiple endocrine neoplasms, neurofibromatosis type 1, medullary neoplasms ( MEN 2) and familiar paraganglioma, thyroid carcinoma, neuroblastoma or pheochromocytoma. ${ }^{2}$ The VHL gene product encodes VHL protein which is responsible for hypoxiainducible factor 1 that leads to hypoxia dependent vascular tumor growth. The novel found VHL mutation in exon 2 is associated with polycythemia but not with cancer. ${ }^{3}$

\section{Case report}

A 31-years old caucasian man came to the neurology emergency department because of the sudden onset of right-sided palsy with sluttered speech. He reported no headache, dizziness or convulsions. After the initial workup, he was admitted to the neurology department. MRI showed subacute ischemic stroke in the posterior part od left insula and operculum. 3D- TOF MR angiography showed occlusion of one of the smaller branches in M2/M3 segment. We performed a doppler ultrasound of carotid and vertebral arteries that indicated vascularized parenchymal structure between ACI and ACE on the right side (Figure 1). Because of that, we did MRI of the neck that verified the inhomogenic structure at the bifurcation of carotid arteries od the right side, dimensions $2,6 \mathrm{X} 1,8 \mathrm{X} 1,9 \mathrm{~cm}$ that resembles paraganglioma (Figure 2).

In the patient's medical history we found erythrocytosis but premorbid history is not well known because he was treated abroad and diagnosed with secondary erythrocytosis due to increased affinity of hemoglobin to oxygen.

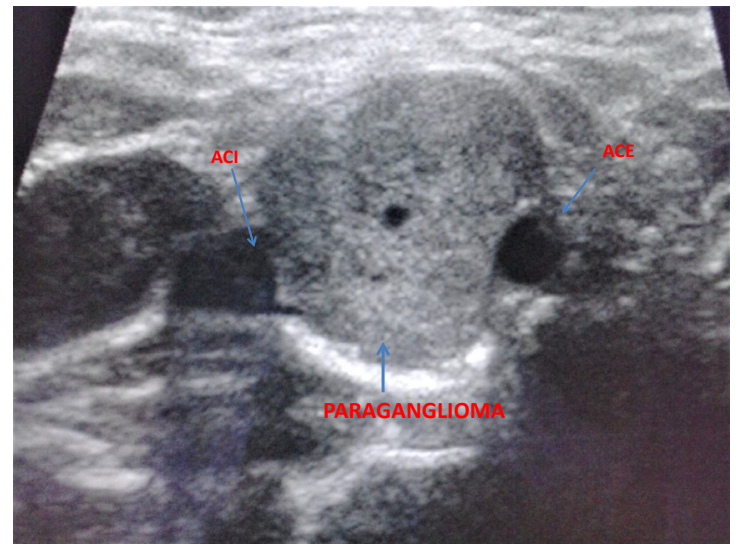

Figure I Ultrasound of the neck- tansversal plane. Arrows are marking internal carotid artery, external carotid artery and paraganglioma.

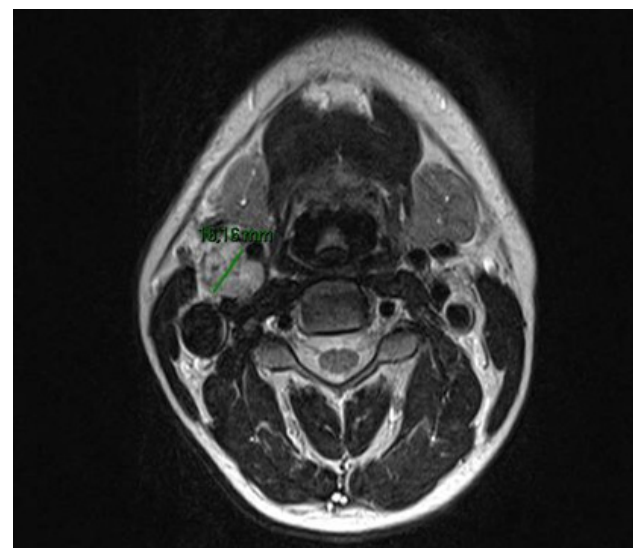

Figure 2 MRI of the patients neck showing large paraganglioma between internal and external carotid arteries. 
Haematologist was consulted and erythrocytapheresis was performed. He was dismissed from hospital without neurological deficit with recommended antiaggregant therapy with ASK 100mg/ day. After dismission he continued with haematologic workup: PET / CT was performed that showed pathological metabolism of F-18 dopa on his neck on both sides and in the abdomen at the aorta bifurcation. Additional diagnostic workup didnt find abnormal hormonal activity (cortisol458nmol/L, chromograninA84,0ug/L, eritropoerin $154 \mathrm{mlU} / \mathrm{L}$, 5-hydroxyindolacetic acid $20 \mathrm{nmol} / \mathrm{dU}$, metanephrine $1.2 \mathrm{umol} / \mathrm{dU}$, normetanephrine $1.7 \mathrm{umol} / \mathrm{dU}$, vanillylmandelic acid $12 \mathrm{umol} / \mathrm{dU}$, hemoglobin variant analysis $22 \mathrm{umol} / \mathrm{dU}$. Other laboratory findings were: E 9,66x10 (12), Hb146g/L, Htc 0,573L/L, MCV 57,9fL, MCH 15,1g/L, Tr 246x10 (9), RTC26/1000, JAK 2V617F was negative.

After that, the suspicion of von Hippel-Lindau syndrome was established. He continued with neck imaging follow-up without progression.

Molecular-genetic testing and sequencing confirmed homozygotic mutation for VHL c.571 C $>\mathrm{G}$ (Croatian form of mutation). ${ }^{4}$

In the meantime, he continued with erythrocytapheresis and went to hematologist abroad for a consultation about paraganglioma on the neck. At the beginning of 2019, the extirpation of paraganglioma of the right side of his neck was performed and for now, he is in regular hematological controls.

\section{Discussion}

Erythrocytosis (and other polycythemia disorders) are well-known risk factors for developing ischemic stroke (not just cerebral). And because of that majority of patients underwent venepunctions (depend on laboratory results) and they all use antiaggregants in their therapy. But sometimes we have to investigate more thural especially when we have very young patients and from geographic area known for specific genetic mutations ${ }^{5}$ and genetic mutations in general because of their risk not only for erythrocytosis and its consequences but because increased risk of developing tumors, ${ }^{1}$ when time is on essence in treating these patients.

After accidentally found paraganglioma on carotid ultrasound we extended our workup and after many years established concrete diagnose.

Mutation phenotype is necessary for establishing disease risk because some paragangliomas may be malignant. ${ }^{2}$ In the majority of cases paraganglioma of the neck are usually benign, non-hormonal secreting tumours, traditionally treated by excision. ${ }^{6}$
Our report emphasizes the importance of genetic testing in young patients with erythrocytosis/polycytosis and not just simply qualify condition as secondary because early diagnosis can prevent unwanted complications and detect eventual tumors on time.

\section{Informed consent}

Written informed consent was obtained from the patient for publication of this case report.

\section{Conflict of interest}

The author declares that there is no conflict of interest.

\section{Acknowledgments}

None.

\section{Funding}

None.

\section{References}

1. Artoni A, Bucciarelli P, Martinelli I. Cerebral thrombosis and myeloproliferative neoplasms. Curr Neurol Neurosci Rep. 2014;14(11):496.

2. Sriphrapradang C, Choopun K, Tunteeratum A, et al. Genotype-Phenotype Correlation in Patients With Germline Mutations of VHL, RET, SDHB, and SDHD Genes: Thai Experience. Clin Med Insights Endocrinol Diabetes. 2017.

3. Lanikova L, Lorenzo F, Yang C, et al. Novel homozygous VHL mutation in exon 2 is associated with congenital polycythemia but not with cancer. Blood. 2013;121(19):3918-3924.

4. Tomasic N, Piterkova L, Huff C, et al. Polycythemia due to Croatian homozygous VHL (571C $>$ G:H191D) mutation has a different phenotype than Chuvash polycythemia (VHL 598C $>$ T:R200W). Haematologica. 2013;98(4):560-567.

5. Pastore Y, Jedlickova K, Guan Y, et al. Mutations of von Hippel-Lindau tumor-suppressor gene and congenital polycythemia. Am J Hum Genet. 2003;73(2):412-419.

6. Harrison L, Corbridge R. Active surveillance management of head and neck paragangliomas: case series and review of the literature. J Laryngol Otol. 2017;131(7):580-584. 